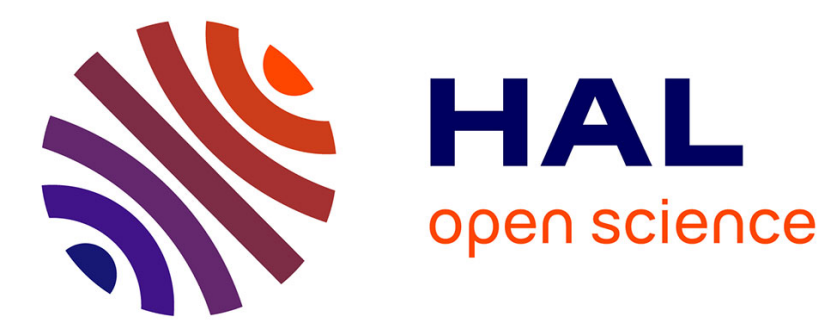

\title{
Estimation of the blood velocity for nanorobotics
}

Matthieu Fruchard, Laurent Arcese, Estelle Courtial

\section{To cite this version:}

Matthieu Fruchard, Laurent Arcese, Estelle Courtial. Estimation of the blood velocity for nanorobotics. IEEE Transactions on Robotics, 2014, 30 (1), pp.93-102. 10.1109/TRO.2013.2288799 . hal-01071275

\section{HAL Id: hal-01071275 \\ https://hal.science/hal-01071275}

Submitted on 3 Oct 2014

HAL is a multi-disciplinary open access archive for the deposit and dissemination of scientific research documents, whether they are published or not. The documents may come from teaching and research institutions in France or abroad, or from public or private research centers.
L'archive ouverte pluridisciplinaire HAL, est destinée au dépôt et à la diffusion de documents scientifiques de niveau recherche, publiés ou non, émanant des établissements d'enseignement et de recherche français ou étrangers, des laboratoires publics ou privés. 


\title{
Estimation of the blood velocity for nanorobotics
}

\author{
Matthieu Fruchard, Laurent Arcese, Estelle Courtial
}

\begin{abstract}
The paper aims at estimating the blood velocity to enhance the navigation of an aggregate in the human vasculature. The considered system is a polymer binded aggregate of ferromagnetic nanorobots immersed in a blood vessel. The drag force depends on the blood velocity and specially acts on the aggregate dynamics. In the design of advanced control laws, the blood velocity is usually assumed to be known or set to a constant mean value to achieve the control objectives. We provide theoretical tools to on-line estimate the blood velocity from the sole measurement of the aggregate position and combine the state estimator with a backstepping control law. Two state estimation approaches are addressed and compared through simulations: a high gain observer and a receding horizon estimator. Simulations illustrate the efficiency of the proposed approach combining online estimation and control for the navigation of an aggregate of nanorobots.
\end{abstract}

Index Terms-Blood velocity, high gain observer, receding horizon estimator, magnetic nanorobots.

\section{INTRODUCTION}

There has been a growing interest in the development of therapeutic microrobots and nanorobots for some years [1]. Such systems have the potential to perform complex surgical procedures without heavy trauma for the patients [2], [3], [4], [5], [6]. Among the numerous prototypes developed, those possessing a deported actuation are the most promising. Indeed these robots do not embed any energy source thus inducing smaller sizes. Untethered robots thus benefit from a possible better therapeutic targeting since they can access to hard-to-reach body's area and also lessen medical side effects linked to surgical operations.

The kind of deported actuators required to control such robots depends on the propulsion strategy. A variable magnetic field is necessary for robots with elastic flagellum [7], [8] or helical flagella [9]. The control of bead pulled robots or swarm of robots [10], [11], [12] is provided by the magnetic field gradients of either Magnetic Resonance Imaging (MRI) devices or magnetic setups (e.g. from Aeon Scientific or Stereotaxis). So as to benefit both from a large motive force in the macrovasculature and from a possible break up in the microvasculature (so as to avoid undesired thrombosis and to improve the targeting), a promising approach is to consider aggregates. Such aggregates of magnetic nanorobots are binded either by a biodegradable ligand [13] or by self-assembly properties [14] as shown on Figure 1.

M. Fruchard and E. Courtial are with PRISME EA 4229, Univ. Orléans, 63 Av de Lattre de Tassigny, F18020, Bourges, France. matthieu.fruchardeuniv-orleans.fr, estelle.courtial@univ-orleans.fr

L. Arcese is with CReSTIC EA 3804, Univ. Reims ChampagneArdenne, Moulin de la Housse, BP 1039, 51687 Reims cedex 2, France. laurent.arceseduniv-reims.fr

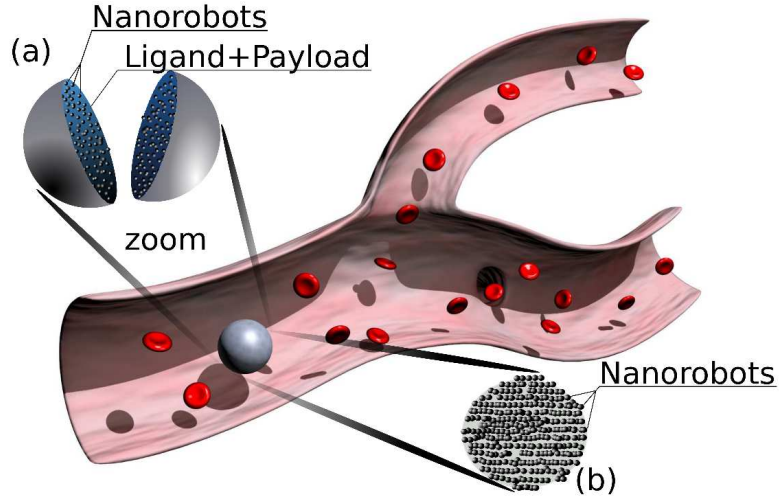

Fig. 1. Aggregate of nanorobots in a blood vessel. (a) Aggregate binded by ligand, including a payload; (b) aggregate binded by magnetic and interaction forces.

Whatever the proposed design, these systems are subject to different forces whom modelling is necessary in order to estimate their dynamic behaviors in a fluidic environment [15], [16]. The blood velocity is usually assumed to be known or set to a constant mean value whilst it is a key nonlinear parameter of the drag force which prevails at a small scale. The measurement of the blood velocity is a difficult task that is often assigned to ultrasonic sensors, at least in vessels close to the sensor. This solution calls for an end-effector servoing so as to track the aggregate position. Another solution relies on a priori knowledge of the blood velocity. Works related to the numerical resolution of the velocity profiles using Computational Fluid Dynamics software had been reported in the literature [17], [18]. But these studies can not be used for real-time purposes because they are based on the Navier Stokes equations whom resolution is computational time consuming. In [15], the authors proposed analytical expressions of the blood velocity profiles. These expressions are valid in the neighborhood of a bifurcation but require an accurate knowledge of the vessel geometry. The blood velocity could also be considered as a disturbance and be rejected in the control law, like in [19] for breathing compensation. However the knowledge of the blood velocity is relevant for the navigation since the main force depends on it. Finally the on-line estimation of the blood velocity seems to be an interesting approach to avoid the drawbacks of the aforementioned methods.

The purpose of this paper is to propose an on-line state estimation of the blood velocity. The latter is modeled by a truncated Fourier series. The model of the nanorobot dynamics taking into account the blood velocity behavior is proved to be observable. Two different state observers are then designed and combined with a backstepping law to achieve a reference 
TABLE I

Physiological Parameters[20], actuation And imaging. X stands for a Compatible device.

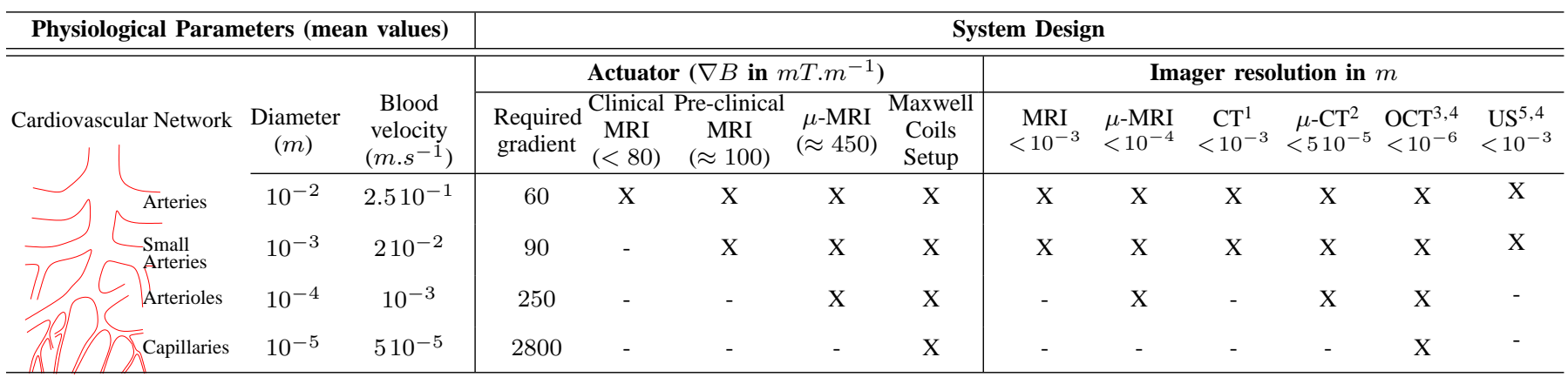

trajectory tracking. The paper consists in five sections. Section II details the need for a blood velocity estimation and proposes a generic blood velocity model. Then, in Section III, the nonlinear model of an aggregate navigating in a blood vessel is presented. Section IV is dedicated to the design of observers: a high gain observer and a receding horizon estimator are addressed to on-line reconstruct the blood velocity. Finally, simulations illustrate the efficiency of the proposed approach combining control and estimation in Section V. Conclusions and discussions on open issues are summarized in Section VI.

\section{Why AND how to ESTIMATE ThE BLOOD VELOCity?}

Magnetically actuated robots are classically described [11] by the motive magnetic force induced by the magnetic field or gradient depending on the robot design [1], the apparent weight and the drag force induced by a non pulsatile blood flow. Hydrodynamic wall effects show that a partial vessel occlusion by the particles results in an optimal ratio between the microrobot and the vessel radii, denoted $r$ and $R$ respectively. As suggested in [14], a first approximation for dimensioning the actuators relies on the study of the optimal ratio of motive over external forces. Table I summarizes these technical constraints and the consequences on the choice of both actuators and imagers.

Optimality requires that the robot goes smaller as it enters smaller vessels. Recently the idea of using microscale carrier made up of a biodegradable aggregate of iron-cobalt nanoparticles and doxorubicin has been rendered possible [13]. In large vessels, a millimeter-sized robot has an important propelling force making possible to resist to the blood drag. In small vessels where the blood flow is lower, a micrometer-sized robot can ensure a sufficient thrust while avoiding any injury caused by embolism or thrombosis. Furthermore the motive force is volumetric whilst the drag force is -at best- surfacic which implies an increasing prevalence of the drag force as the vessel radius decreases. Among the different forces affecting the robot navigation in

\footnotetext{
${ }^{1} \mathrm{CT}$ stands for Computed Tomography device

${ }^{2}$ Cannot be used on living animals

${ }^{3}$ OCT stands for Optical Coherence Tomography device

${ }^{4}$ Limited penetration depth

${ }^{5}$ US stands for Ultrasound device
}

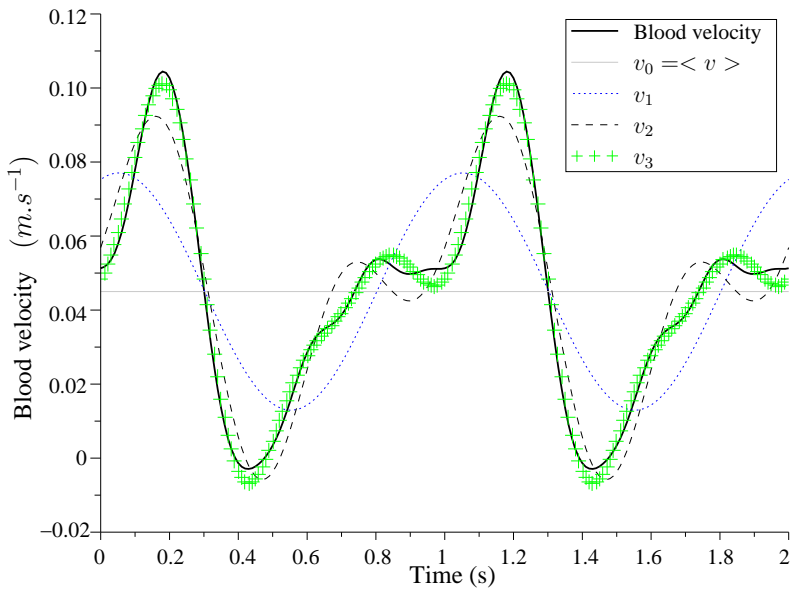

Fig. 2. Blood velocity $v$ (black solid line), mean value $v_{0}$ (gray solid line) and $n$th order truncated Fourier series $v_{n}: v_{1}$ (blue dots), $v_{2}$ (black dashes) and $v_{3}$ (green crosses).

the vasculature, the most disturbed by external time-varying perturbations is the drag force because of the pulsatile blood flow. Several possibilities to deal with this periodic perturbation might be considered: direct measurement, disturbance rejection or blood velocity estimation.

Physiological periodic perturbations are usual issues in medical robotics and are often addressed using periodic signal rejection: either using filtering of the output or using a precompensation in the control law, like in [19] for breathing rejection. For the present case, both solutions require the blood velocity measurement. However the pulsatile blood velocity is a local information and is consequently more difficult to be measured. Studies have been carried out to measure the blood velocity using the Doppler effect with ultrasound or MRI devices [21], [22]. The quality of the measurement depends on the ultrasound transducer orientation during the acquisition and it would require to ensure the servoing of the devoted imager on the robot position as it navigates through the human body. When the robot penetrates deeper into the human body, the device resolution, as well as the perturbations induced by the different tissues separating the robot from the sensor, may impact the measurement reliability. Finally the poor spatial accuracy of such measurements is not compatible with the precision required to discriminate the parabolic 
blood velocity profile; yet the robot does not face the same drag depending on its position in the vessel. The proposed approach is different since it does not rely on the direct measurement of this velocity but on the disturbance estimation.

Since an imager is already necessary to localize the robot and thus provides an output of the system, one can exploit a model of the system to reconstruct unmeasured states. Observers of the robot velocity have already been studied in [23]. We propose to extend this approach to estimate the blood velocity. It requires to have a dynamical model of the blood velocity behavior. Arterial pulsatile flow profiles are usually modeled using the Womersley model [24], which results in a truncated Fourier series as shown on Fig. 2. It is easy to show that any velocity $\aleph_{1}$ expressed as a second-order truncated Fourier series is a solution of the autonomous system:

$$
\left(\mathcal{S}_{\aleph_{1}}\right) \quad\left\{\begin{array}{l}
\dot{\aleph}_{1}=\aleph_{2} \\
\dot{\aleph}_{2}=-\omega^{2}\left(\aleph_{1}-\aleph_{3}\right) \\
\dot{\aleph}_{3}=0
\end{array}\right.
$$

where $\aleph_{3}$ is the average value of the blood velocity and $\omega$ is the pulsation of the blood flow assumed to be known. For a blood velocity $\aleph_{1}$ defined as a $n$th order truncated Fourier series, (1) can be extended in the $(2 n+1)$-dimensional form:

$$
\left(\mathcal{S}_{\aleph}\right) \quad\left\{\begin{array}{cl}
\dot{\aleph}_{1} & =\aleph_{2} \\
\dot{\aleph}_{2} & =-\omega^{2}\left(\aleph_{1}-\aleph_{3}\right) \\
\vdots & \\
\dot{\aleph}_{2 k-1} & =\aleph_{2 k} \\
\dot{\aleph}_{2 k} & =-\omega^{2}\left(k^{2} \aleph_{2 k-1}-\aleph_{2 k+1}\right) \\
\vdots & \\
\dot{\aleph}_{2 n+1} & =0
\end{array}\right.
$$

$\forall k \in\{1, \ldots, n\}$ and where the mean value is $\aleph_{2 n+1}$, up to a constant factor. See the Appendix for technical details.

The objectif is then to link the disturbance dynamic extension (2) to the robot modeling.

\section{MODELING}

Let us consider an aggregate navigating in the arterial network. Any system immersed in a moving fluid is subjected to two forces: the drag force and the buoyancy force. This section is devoted to briefly introduce these forces and the magnetic force that controls the robot along a pre-planned reference trajectory (see e.g. [15] for more details). The internal forces affecting the aggregate cohesion are not taken into account.

\section{A. Forces}

1) Hydrodynamic drag force: In a fluidic environment, any system is subjected to the drag force which opposes its motion:

$$
\left\{\begin{aligned}
\vec{F}_{d} & =-\frac{1}{2} \rho_{f} A C_{d}\left(\frac{\|\vec{v}\|}{\beta}\right)^{2} \frac{\vec{v}}{\|\vec{v}\|} \\
C_{d} & =\frac{24}{R e}+\frac{6}{1+\sqrt{R e}}+0.4 \\
R e & =\frac{2 r \rho_{f}\|\vec{v}\|}{\beta \eta}
\end{aligned}\right.
$$

where $A$ is the frontal area, the drag coefficient $C_{d}$ is given by [25], $\beta$ is a dimensionless ratio related to the wall effect caused by the vessel occlusion by a spherical aggregate of radius $r$ [26], and $v$ denotes the relative velocity between the aggregate and the fluid. $\eta$ and $\rho_{f}$ denote respectively the blood viscosity and density. In the case of a spherical aggregate, the drag force can be rewritten as:

$$
\vec{F}_{d}=-\left(a|v|+b v^{2}+c \frac{v^{2}}{1+d \sqrt{|v|}}\right) \frac{\vec{v}}{\|\vec{v}\|}
$$

The parameters in (4) are inherited from (3):

$$
\left\{\begin{aligned}
a & =\frac{6 \pi \eta r}{\beta}, \quad b=\frac{0.2 \rho_{f} \pi r^{2}}{\beta^{2}} \\
c & =\frac{3 \rho_{f} \pi r^{2}}{\beta^{2}}, \quad d=\sqrt{\frac{2 r \rho_{f}}{\beta \eta}}
\end{aligned}\right.
$$

2) Magnetic force: The propulsion of the magnetic robot is provided by the magnetic force $\vec{F}_{m}$ induced by the magnetic field gradients. This force can be written as:

$$
\vec{F}_{m}=\tau_{m} V(\vec{M} \cdot \nabla) \vec{B}
$$

where $V$ is the aggregate volume, $\vec{B}$ the magnetic field, $\vec{M}$ the magnetization of the nanorobots and $\tau_{m}=V_{m} / V$ the ferromagnetic ratio with $V_{m}$ the ferromagnetic volume.

3) Apparent weight: The apparent weight of the aggregate results from the contribution of the weight and the buoyancy:

$$
\vec{W}_{a}=V\left(\rho-\rho_{f}\right) \vec{g}
$$

with the aggregate density $\rho=m / V$ where $m$ is the aggregate mass. Since the aggregate is made of ferromagnetic nanorobots and a payload (ligand, drugs, micelle...), the aggregate density can be decomposed as $\rho=\tau_{m} \rho_{m}+\left(1-\tau_{m}\right) \rho_{p}$ where $\rho_{m}$ and $\rho_{p}$ are respectively the density of the magnetic nanorobots and the density of the payload.

\section{B. State Space Representation}

Let us consider the system derived from (4), (6) and (7):

$$
\left(\mathcal{S}_{1}\right) \quad\left\{\begin{aligned}
\dot{p}_{1} & =p_{2} \\
\dot{p}_{2} & =f\left(p_{2}, \aleph_{1}\right)+\frac{\tau_{m} M}{\rho} u \\
y & =p_{1}
\end{aligned}\right.
$$

where $p_{1}$ and $p_{2}$ denote respectively the aggregate position and velocity, $u$ the control input and $\aleph_{1}$ the blood velocity. The output $y$ is the position of the aggregate measured by the imager. The expression of the function $f\left(p_{2}, \aleph_{1}\right)$ is given by:

$$
\begin{aligned}
f\left(p_{2}, \aleph_{1}\right)= & {\left[-\sigma\left(p_{2}-\aleph_{1}\right)\left(a\left|p_{2}-\aleph_{1}\right|+b\left(p_{2}-\aleph_{1}\right)^{2}\right.\right.} \\
& \left.\left.+c \frac{\left(p_{2}-\aleph_{1}\right)^{2}}{1+d \sqrt{\left|p_{2}-\aleph_{1}\right|}}\right)+V\left(\rho_{f}-\rho\right) g\right] \frac{1}{m},
\end{aligned}
$$

where the sign function $\sigma$ is defined by:

$$
\sigma(\xi)=\left\{\begin{array}{cll}
-1 & \text { if } & \xi<0 \\
0 & \text { if } & \xi=0 \\
1 & \text { if } & \xi>0
\end{array}\right.
$$

The pulsatile periodic blood velocity is modeled by a $n$th order truncated Fourier series given by the system (2). Note that $\aleph$ 
belongs to a compact set $\mathcal{K}_{\aleph}$. Let $x=\left[p^{T}, \aleph^{T}\right]^{T} \in \mathbb{R}^{2 n+3}$ denote an extended state vector. From (2) and (8), we obtain an extended system in the single output control-affine form $\dot{x}=F(x)+G u, y=h(x), \forall k \in\{1, \ldots, n\}$ :

$$
\left\{\begin{array}{cl}
\dot{x}_{1} & =x_{2} \\
\dot{x}_{2} & =f\left(x_{2}, x_{3}\right)+\frac{\tau_{m} M}{\rho} u \\
\vdots & \\
\dot{x}_{2 k+1} & =x_{2 k+2} \\
\dot{x}_{2 k+2} & =-\omega^{2}\left(k^{2} x_{2 k+1}-x_{2 k+3}\right) \\
\vdots & \\
\dot{x}_{2 n+3} & =0 \\
y & =h(x)=x_{1} .
\end{array}\right.
$$

In order to implement a feedback control law, the state vector of the system (11) is required to be known. Given unmeasured variables, a state-observer has to be designed. The next section is devoted to the synthesis of observers for estimating the blood velocity.

\section{Main Results}

The controllability and the design of backstepping control laws used to Lyapunov stabilize the system (8) have been addressed in [23]. We briefly recap these results to point out the necessity of estimating both the unmeasured robot and blood velocities which are required for implementing the control law. We then address the main results of the paper: the observability of the extended system (11) and the design of two classes of observers using either a high gain observer or a receding horizon estimator.

\section{A. Prerequisites}

Proposition 1 Let $X_{\text {ref }}=\left[X_{r}(t), \dot{X}_{r}(t), \ddot{X}_{r}(t)\right]$ denote any continuous and bounded reference trajectory. The system (8) is locally controllable along the reference trajectory.

Proof: The controllability of system (8) is inherited from the controllability of its linearized time-variant system along the reference trajectory $X_{r e f}(t)$ [27], [28]. See [23] for more details.

Proposition 2 Let $\theta$ denote any linear uncertain parameter in (9) such that $f\left(p_{2}, \aleph_{1}\right)=f_{0}\left(p 2, \aleph_{1}\right)+\varphi\left(p_{2}, \aleph_{1}\right) \theta$. Under assumptions of Proposition 1 and for $\aleph_{1}$ bounded, the adaptive backstepping control law

$\left\{\begin{array}{c}u=\frac{\rho}{\tau_{m} M}\left[-\left(k_{1}+k_{2}\right)\left(p_{2}-\dot{X}_{r}\right)-\left(1+k_{1} k_{2}\right)\left(p_{1}-X_{r}\right)\right. \\ \left.\quad-f_{0}\left(p_{2}, \aleph_{1}\right)-\varphi\left(p_{2}, \aleph_{1}\right) \hat{\theta}+\ddot{X}_{r}\right] \\ \dot{\hat{\theta}}=\Gamma \varphi^{T}\left(p_{2}, \aleph_{1}\right)\left[\left(p_{2}-\dot{X}_{r}\right)+k_{1}\left(p_{1}-X_{r}\right)\right]\end{array}\right.$

stabilizes the system (8) along any $\mathcal{C}^{0}$ reference trajectory for any initial estimate $\hat{\theta}(0)$ with the controller gains $k_{1}, k_{2}>0$ and a positive-definite matrix gain $\Gamma$.

Proof: The proof, given in [23], relies on a two steps constructive proof of the Lyapunov stabilizing controller.

If no uncertain parameter is considered, Proposition 2 stands with $f_{0}=f$ and no updating that is $\dot{\hat{\theta}}=0$. In every instance, the control law (12) needs to know the position and velocity of the robot and the velocity of the blood. The former, i.e. the position, is measured by the imaging device whereas the velocities are not and consequently require to be estimated.

\section{B. Observability of the Extended System}

Lemma 1 Let $\mathcal{K}$ denote any compact subset of a neighborhood of $\left(X_{r}, \dot{X}_{r}\right) . \forall x \in \mathcal{K} \times \mathcal{K}_{\aleph}$, the vector field $F$ satisfies the following properties:

P1) $\forall i \in\{1, \ldots, 2 n+1\}, \forall j \geq 2, \frac{\partial F_{i}(x)}{\partial x_{i+j}}=0$;

P2) $\forall i \in\{1, \ldots, 2 n+2\}, \exists \alpha_{i}, \beta_{i}>0: \alpha_{i} \leq \frac{\partial F_{i}(x)}{\partial x_{i+1}} \leq \beta_{i}$;

P3) $\forall i \in\{1, \ldots, 2 n+3\}, \exists \gamma_{i} \geq 0:\left|\frac{\partial F_{i}(x)}{\partial x_{j}}\right| \leq \gamma_{i}$, $\forall j \leq i+1$.

Proof: From (11), it is straightforward that $(\mathbf{P} 1)$ is satisfied.

The property $(\mathbf{P} 2)$ is easily obtained for the linear part of the system i.e. for $i \neq 2$ with

$$
\left\{\begin{aligned}
\alpha_{2 k+1} & =\beta_{2 k+1}=1, & & \forall k \in\{0, \ldots n\} \\
\alpha_{2 k+2} & =\beta_{2 k+2}=\omega^{2}, & & \forall k \in\{1, \ldots n\} .
\end{aligned}\right.
$$

$\alpha_{2}$ and $\beta_{2}$ are detailed hereafter. We have the following even function by differentiating the expression in (11) and setting $v=x_{2}-x_{3}$ :

$$
\frac{\partial F_{2}(v)}{\partial x_{3}}=\left[a+b|v|+2 c \frac{|v|}{1+d \sqrt{|v|}}-\frac{c d|v|^{\frac{3}{2}}}{2(1+d \sqrt{|v|})^{2}}\right] \frac{1}{m} .
$$

We consequently have $\frac{\partial F_{2}(v)}{\partial x_{3}}=a / m$ equivalent to

$$
|v|\left(2 b(1+d \sqrt{|v|})^{2}+2 c(1+d \sqrt{|v|})-\frac{c d \sqrt{|v|}}{2}\right)=0,
$$

whose solutions are

$$
\left\{\begin{array}{l}
v=0 \\
|v|=\frac{\frac{3 c}{2} \sqrt{1-\frac{16 b}{9 c}}-4 b-\frac{3 c}{2}}{2 b d} .
\end{array}\right.
$$

Since we have, from (5), $c>\frac{16 b}{9}$, it is not difficult to show that $\frac{\partial F_{2}(v)}{\partial x_{3}}>a / m, \forall v \neq 0$. Hence we obtain $\alpha_{2}=a / m$.

The upper bound $\beta_{2}=\max _{x \in \mathcal{K}} \frac{\partial F_{2}(x)}{\partial x_{3}}$ is inherited from the continuous differentiability of $\frac{\partial F_{2}(v)}{\partial x_{3}}$ on $\mathbb{R}^{*}$ which implies its local Lipschitz continuity. The property $(\mathbf{P} 2)$ is consequently satisfied $\forall i \in\{1, \ldots, 2 n+2\}$.

The property (P3) is easily obtained for the linear part of the system:

$$
\left\{\begin{array}{lll}
\gamma_{2 k+1}=0, & \forall k \in\{0, \ldots n\} \\
\gamma_{2 k+2}=\omega^{2} k^{2}, & \forall k \in\{1, \ldots n\} .
\end{array}\right.
$$

Using the previous result and noticing that $\frac{\partial F_{2}(x)}{\partial x_{2}}=-\frac{\partial F_{2}(x)}{\partial x_{3}}$, the property (P3) is satisfied with $\gamma_{2}=\beta_{2}$. 
Remark 1 The property $(\boldsymbol{P} 1)$ is related to the triangular structure of the system. The existence of constants $\beta_{i}$ and $\gamma_{i}$ in $(\boldsymbol{P} 2)$ and $(\boldsymbol{P} 3)$ are linked to the Lipschitz property of functions $F_{i}$, at least locally. In this case, one can use globally Lipschitz extensions of the $\mathcal{C}^{1}$ functions $F_{i}$.

Proposition 3 The system (11) is uniformly observable.

Proof: The observability matrix is

$$
\mathcal{O}=\frac{\partial L_{F}^{i} h(x)}{\partial x_{j}}, \quad 0 \leq i \leq 2 n+2,1 \leq j \leq 2 n+3
$$

where $L_{F}^{i} h(x)$ denotes the $i$ th Lie derivative of the output $h(x)$ of the system (11) along the vector field $F$. Lie differentiating the output of the system (11), we obtain, $\forall k \in\{1, \ldots, n\}$ :

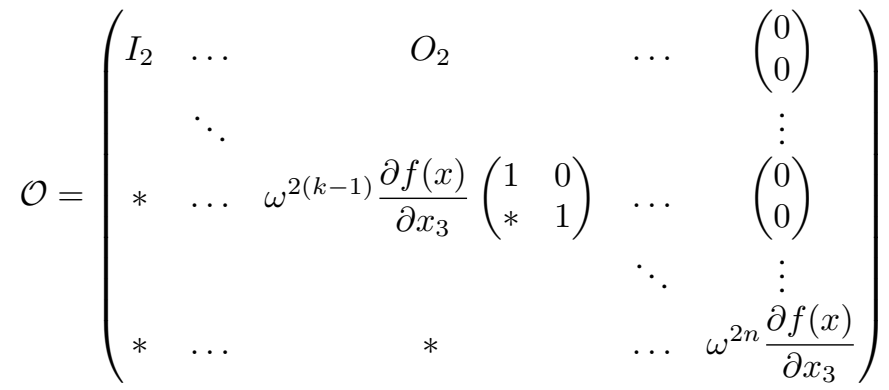

where $*$ stands for bounded functions. It is straightforward that the observability matrix is full ranked as $\frac{\partial f(x)}{\partial x_{3}} \neq 0$ from the property (P2) of Lemma 1, hence the result.

Since it is possible to estimate the full state vector of the extended system (11) using the sole output accessible to measurement, observers for the system (11) can be designed. Two different kinds of observers are studied in the next sections: a high gain observer and a receding horizon estimator. The first one is theoretically well founded and the second one is well known for its easy implementation.

\section{High Gain Observer}

Since the vector fields are locally Lipschitz continuous, high gain observers are a promising tool. Indeed, we have already addressed high gain observers in [23] for system (8) which can easily be written in the observability canonical form [29]. Yet writing system (11) in the observability canonical form requires an exact linearization feedback which is very sensitive to output noise. To avoid this drawback, we would consequently rather develop a high gain observer that directly exploits the triangular structure [30] -related to property (P1)of the system (11).

Proposition 4 Let $\mathcal{K}$ denote any compact subset of a neighborhood of $\left(X_{r}, \dot{X}_{r}\right)$ and $\mathcal{U}$ the compact set of admissible control inputs. Then $\forall x(0) \in \mathcal{K} \times \mathcal{K}_{\aleph}, \forall \hat{x}(0) \in \mathcal{K} \times \mathcal{K}_{\aleph}$, $\forall u \in \mathcal{U}, \forall \hat{\theta}(0), \exists L_{0}>1$ such that $\forall L>L_{0}$,

$$
\dot{\hat{x}}=F(y, \hat{x}, \hat{\theta})+G u-\delta \Delta_{\epsilon} \Delta S^{-1} C^{T}\left(C \Delta_{\epsilon}^{-1} \hat{x}-y\right)
$$

is a high gain observer for system (11) on the compact set $\mathcal{K} \times \mathcal{K}_{\aleph}$ where $\Delta=\operatorname{diag}\left(L, \ldots, L^{2 n+3}\right)$ and
$\Delta_{\epsilon}=\operatorname{diag}\left(\epsilon_{1}, \ldots, \epsilon_{2 n+3}\right)$ respectively denote the gain and a normalization diagonal matrix. $S$ is a tridiagonal symmetric definite positive matrix such that the following Lyapunov inequality is satisfied:

$$
A^{T}(t) S+S A(t)-\delta_{0} C^{T} C \leq \mu I, \forall t \geq 0
$$

with $\delta_{0}>0, \mu\left(\delta_{0}\right)>0$, the parameter $\delta \geq \frac{\delta_{0}}{2}$ and the matrix $A(t)$ which nonzero entries are only $a_{i, i+1}(t) \in$ $\left[\frac{\alpha_{i} \epsilon_{i+1}}{\epsilon_{i}}, \frac{\beta_{i} \epsilon_{i+1}}{\epsilon_{i}}\right], \forall i \leq 2 n+2$.

Proof: We first perform a normalization step by defining a new state vector $z=\Delta_{\epsilon}^{-1} x$ where the choice of the nonzero parameters $\epsilon_{i}$ is related to both the conditioning matrix $S$ in (20) and the resulting minimal gain $L_{0}$. In the sequel we consider the $z$-system $\dot{z}=F_{z}(z, t)+G_{z} u$ derived from (11): $\forall k \in\{1, \ldots, n\}$

$$
\left\{\begin{array}{cl}
\dot{z}_{1} & =\frac{\epsilon_{2}}{\epsilon_{1}} z_{2} \\
\dot{z}_{2} & =f_{z}\left(z_{2}, z_{3}, t\right)+\frac{\tau_{m} M}{\rho \epsilon_{2}} u \\
\vdots & \\
\dot{z}_{2 k+1} & =\frac{\epsilon_{2 k+2}}{\epsilon_{2 k+1}} z_{2 k+2} \\
\dot{z}_{2 k+2} & =\frac{\omega^{2} \epsilon_{2 k+3}}{\epsilon_{2 k+2}} z_{2 k+3}-\frac{\omega^{2} k^{2} \epsilon_{2 k+1}}{\epsilon_{2 k+2}} z_{2 k+1} \\
\vdots & \\
\dot{z}_{2 n+3} & =0
\end{array}\right.
$$

with $f_{z}\left(z_{2}, z_{3}, t\right)=\frac{1}{\epsilon_{2}} f\left(\epsilon_{2} z_{2}, \epsilon_{3} z_{3}, \hat{\theta}\right)$.

Let $e=\hat{z}-z$ denote the observation error. Using (21) and (19), we obtain:

$$
\dot{e}=F_{z}(\hat{z}, t)-F_{z}(z, t)-\delta \Delta S^{-1} C^{T} C e .
$$

The basic idea of the proof is to decompose the difference $F_{z}(\hat{z}, t)-F_{z}(z, t)$ as follows:

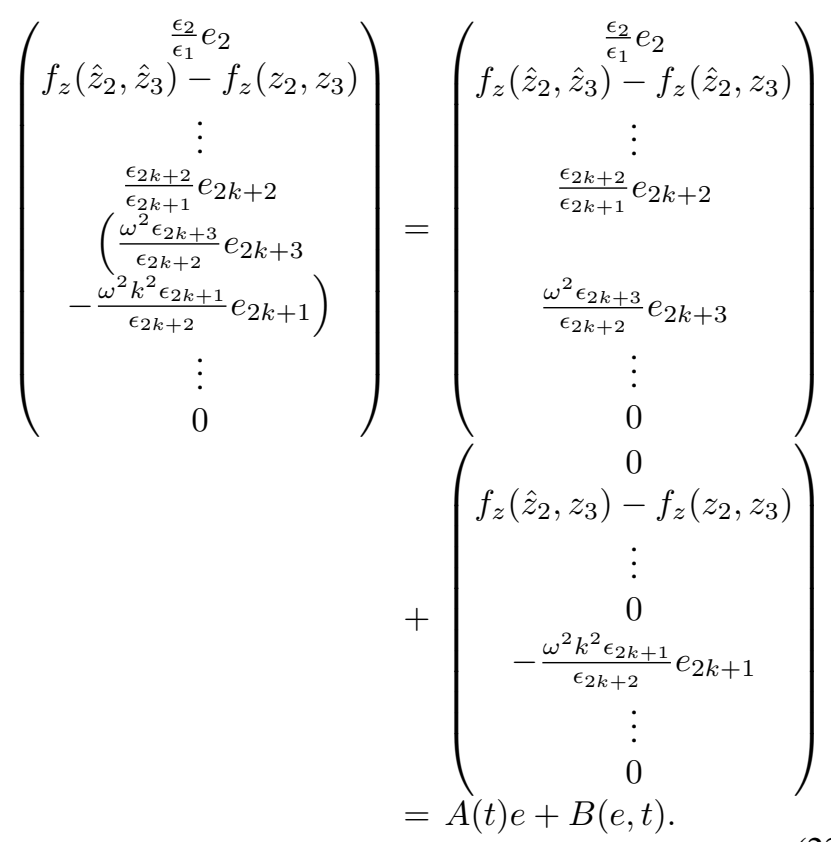


Using the mean value theorem, we get the matrix $A$ defined in Proposition 4 with

$$
a_{i, i+1}(t)=\frac{\epsilon_{i+1}}{\epsilon_{i}} \frac{\partial F_{z, i}}{\partial z_{i+1}}\left(\hat{z}_{1}, \ldots, \hat{z}_{i}, z_{i+1}+\phi(t) e_{i+1}, t\right)
$$

for some (unknown) $\phi(t) \in[0,1]$ and the vector $B(e, t)$ whose nonzero components are even ones:

$$
\left\{\begin{aligned}
b_{2}(t) & =\frac{\partial F_{z, 2}}{\epsilon_{2} \partial z_{2}}\left(\hat{z}_{1}, z_{2}+\phi(t) e_{2}, z_{3}, t\right) \\
b_{2 k+2} & =-\frac{\omega^{2} k^{2} \epsilon_{2 k+1}}{\epsilon_{2 k+2}}, \forall k \in\{1, \ldots, n\} .
\end{aligned}\right.
$$

Bounds on $a_{i, i+1}(t)$ and on $b_{2}(t) \leq \gamma_{2}$ are inherited from properties $(\mathbf{P} 2)$ and $(\mathbf{P} 3)$ of Lemma 1 .

The usual change of coordinates $\varepsilon_{i}=\frac{e_{i}}{L^{i}}$ leads to:

$$
\dot{\varepsilon}=L\left(A(t)-\delta S^{-1} C^{T} C\right) \varepsilon+D(t) \varepsilon
$$

where the nonzero entries of the matrix $D(t)$ are:

$$
d_{2,2}(t)=b_{2}(t), \quad d_{2 k+2,2 k+1}=b_{2 k+2} / L \quad \forall k \in\{1, \ldots, n\} .
$$

The following upper bounds:

$$
\left\{\begin{array}{l}
\left|d_{2,2}(t)\right| \leq \gamma_{2} \\
\left|d_{2 k+2,2 k+1}\right| \leq \frac{\omega^{2} k^{2} \epsilon_{2 k+1}}{\epsilon_{2 k+2}}=\frac{\gamma_{2 k+2} \epsilon_{2 k+1}}{\epsilon_{2 k+2}}
\end{array}\right.
$$

are satisfied since $L \geq 1$.

A candidate Lyapunov function can be given by:

$$
V(t)=\varepsilon^{T} S \varepsilon \leq \lambda\|\varepsilon\|^{2} .
$$

Differentiating (29) using (26) leads to:

$$
\dot{V}(t)=L \varepsilon^{T}\left(A^{T} S+S A-2 \delta C^{T} C\right) \varepsilon+2 \varepsilon^{T} S D \varepsilon .
$$

We recap the Lemma of [30]: assuming that property (P2) is satisfied, then $\forall \delta_{0}>0, \exists \mu>0$ and $\exists S$ a tridiagonal positive definite matrix such that (20) holds. Using (20) in (30), we obtain:

$$
\dot{V}(t) \leq-\mu L\|\varepsilon\|^{2}-\left(2 \delta-\delta_{0}\right) L\|C \varepsilon\|^{2}+2 \varepsilon^{T} S D \varepsilon .
$$

Set $2 \delta \geq \delta_{0}$ leads to

$$
\dot{V}(t) \leq-\mu L\|\varepsilon\|^{2}+2 \varepsilon^{T} S D(t) \varepsilon .
$$

Set $M(\gamma, S, \epsilon)=\|S D(t)\|_{\infty}$ involves

$$
\dot{V}(t) \leq-(\mu L-2 M)\|\varepsilon\|^{2} \leq-(\mu L-2 M) \frac{V(t)}{\lambda}
$$

with $\lambda$ the highest eigenvalue of $S$. Let $L_{0}=\max \left(1, \frac{2 M}{\mu}\right)$, it follows from (33) that $\forall L>L_{0}$, (19) is an exponential observer for system (11).
TABLE II

PARAMETER VALUES

\begin{tabular}{|c|c|c|}
\hline Blood viscosity & $\eta$ & $16 \times 10^{-3}[$ Pa.s $]$ \\
\hline Blood density & $\rho_{f}$ & $1060\left[\mathrm{~kg} \cdot \mathrm{m}^{-3}\right]$ \\
\hline Nanorobot density & $\rho_{m}$ & $7500\left[\mathrm{~kg} \cdot \mathrm{m}^{-3}\right]$ \\
\hline Aggregate radius & $r$ & $2.510^{-4}[\mathrm{~m}]$ \\
\hline Vessel diameter & $D$ & $310^{-3}[\mathrm{~m}]$ \\
\hline Payload density & $\rho_{p}$ & $1500\left[\mathrm{~kg} \cdot \mathrm{m}^{-3}\right]$ \\
\hline Ferromagnetic ratio & $\tau_{m}$ & 0.75 \\
\hline Magnetization & $M$ & $1.23 \times 10^{6}[\mathrm{A.m}$ \\
\hline Controller gains & $\left(k_{1}, k_{2}\right)$ & $(7,14)$ \\
\hline High gain & $L$ & 3 \\
\hline
\end{tabular}

TABLE III

INITIAL CONDITIONS FOR THE SYSTEM AND THE OBSERVER

\begin{tabular}{|c|c|}
\hline$\left(x_{1}, x_{2}, x_{3}, x_{4}, x_{5}\right)$ & $(0 ; 0 ; 0.1075 ; 0 ; 0.05)$ \\
\hline$\left(\hat{x}_{1}, \hat{x}_{2}, \hat{x}_{3}, \hat{x}_{4}, \hat{x}_{5}\right)$ & $(0.001 ; 0.01 ; 0 ; 0.001 ; 0.01)$ \\
\hline
\end{tabular}

\section{Receding Horizon Estimation (RHE)}

Among the different approaches of state estimation, RHE presents an attractive alternative to the theoretically wellfounded observers by proposing a systematic way to design an optimization-based observer [31]. The estimation is formulated as solving on-line a nonlinear optimization problem. The principle of RHE is to minimize a cost function over a past finite time interval, usually named estimation horizon and denoted $N_{e}$. The difference between the measured output $y$ and the estimated output $\hat{y}$ over the estimation horizon defines the cost function $J$. Due to the sampled measurements (with $T_{e}$ the sampling period), the state estimation problem is written in discrete-time. At the current time $k$, the function $J$ is minimized with respect to the state $x$ at time $\left(k-N_{e}\right)$. The estimated output $\hat{y}(k)$ is then computed thanks to the model and the computed optimal state estimate $\hat{x}^{\star}\left(k-N_{e}\right)$. At each sampling time, the past estimation horizon moves one step forward and the whole procedure (model-based estimation and optimization) is repeated to guarantee the robustness of the approach in regard to disturbances and model mismatches. The mathematical formulation of the RHE is given by:

$$
\min _{x\left(k-N_{e}\right)} J(x)=\sum_{j=k-N_{e}}^{k}[y(j)-\hat{y}(j)]^{T} Q[y(j)-\hat{y}(j)]
$$

subject to:

$$
\left\{\begin{array}{l}
\hat{x}(k+1)=F_{k}(\hat{x}(k))+G_{k} u(k) \\
\hat{y}(k)=h_{k}(\hat{x}(k)) .
\end{array}\right.
$$

Q is a symmetric definite positive matrix and the nonlinear model (35) obtained by a second-order Runge-Kutta method describes the dynamics of the process (11) in discrete time.

The main advantages of RHE are its capability to explicitly take into account state constraints, its easy implementation requiring the setting of two parameters $\left(N_{e}, Q\right)$ and its robustness in regard to disturbances because of the repeated optimization procedure. On the other hand, it suffers from a lack of convergence proof since the solution is numerical. 

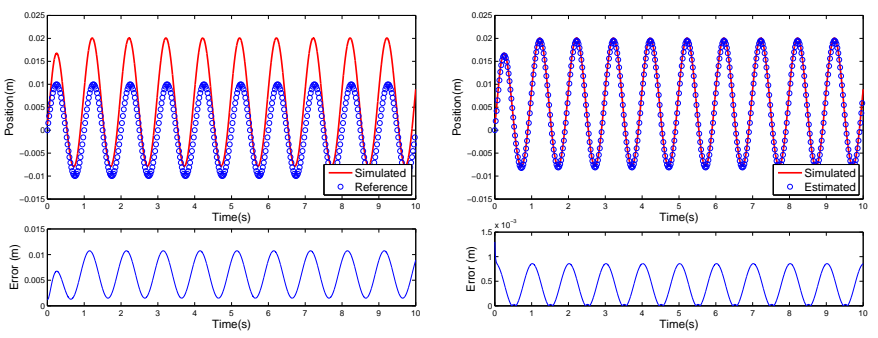

(a) Aggregate position: reference and real trajectories and tracking error.

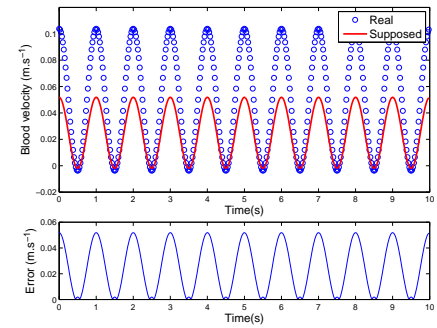

(c) Blood velocity: real and estimated velocities and estimation error. (b) Aggregate position: real and estimated trajectories and estimation error.

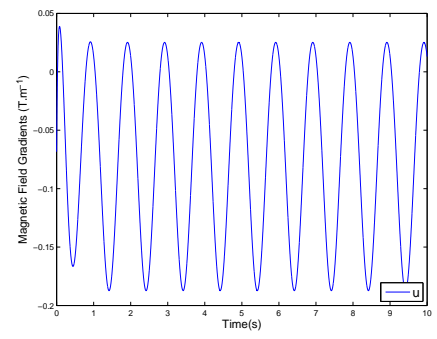

(d) Control input: $\nabla B$.
Fig. 3. Simulation without an estimation of the blood velocity.

\section{Simulations}

The first simulation illustrates that the tracking is highly degraded when an incorrect a priori knowledge of the blood velocity is considered, and consequently emphasizes the need for an estimation of the blood velocity. In the two last simulations the aggregate position is assumed to be measured within an accuracy of $100 \mu m$ consistent with the $\mu$-MRI resolution. A Gaussian white noise with a standard deviation of $100 \mu \mathrm{m}$ is thus added to the measured output. The nominal values of parameters are given in Table II and the initial conditions of the system and the observer in Table III. Without loss of generality, the dynamic of the blood velocity is modeled by a second-order truncated Fourier series.

\section{A. Simulation without an estimation of the blood velocity}

In this simulation, we show that an error on the blood velocity estimation particularly affects the tracking. The simulation is performed assuming a $50 \%$ error on the blood velocity with respect to its nominal value (see Figure 3(c)). Figure 3(a) show that the tracking is degraded because of a wrong estimation of the blood velocity. One can notice a tracking error greater than $1 \mathrm{~cm}$ and a maximum position estimation error about $1 \mathrm{~mm}$ on the Figure 3(c). Although the control input ensures the stability of the system (see Figure 3(d)), the position error is too large and compromises the access to a specific area of the human body. The interest of estimating the blood velocity is demonstrated in the two next simulations.
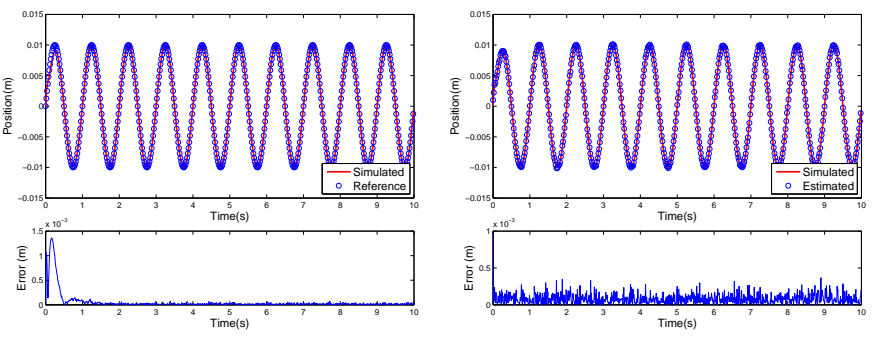

(a) Aggregate position: reference and (b) Aggregate position: real and estireal trajectories and tracking error. mated trajectories and estimation error.

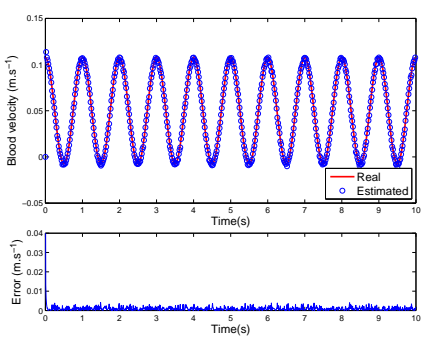

(c) Blood velocity: real and estimated velocities and estimation error.

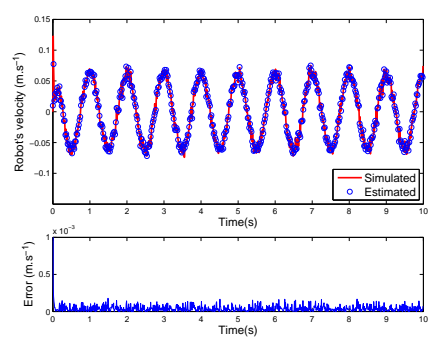

(d) Aggregate velocity: real and estimated velocities and estimation error.

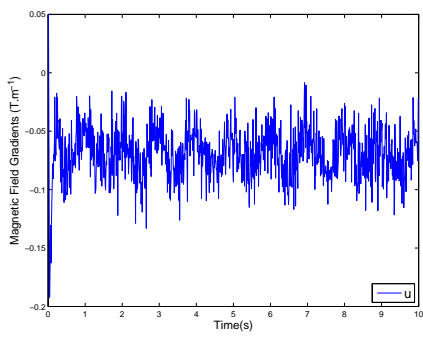

(e) Control input: $\nabla B$.

Fig. 4. Simulation with a high gain observer.

\section{B. Simulation with a high gain observer}

The estimation of the full state vector is performed by a high gain observer with parameters $\delta=2500$ and $S$ given by:

$$
S=\left(\begin{array}{ccccc}
0.13 & -0.015 & 0 & 0 & 0 \\
-0.015 & 0.01 & -0.022 & 0 & 0 \\
0 & -0.022 & 0.1 & -1.5 & 0 \\
0 & 0 & -1.5 & 100 & -50 \\
0 & 0 & 0 & -50 & 60
\end{array}\right)
$$

The aim of the observer is twofold. First, it reconstructs the aggregate velocity needed to compute the control inputs. The control input, based on Lyapunov functions, ensures the stability of the system along the pre-planned reference trajectory. Second, the observer on-line estimates the blood velocity in order to compensate for modeling errors or incorrect a priori knowledge that affects the drag force.

Figure 4(a) illustrates that the tracking of the reference trajectory by the aggregate is efficient and the system is stable. One can notice a transient phase between $t=0 s$ and $t=1 s$ due to the different initial conditions (see Table III). Figures 4(b), 4(c) and 4(d) show that the convergence of the observer is not affected by the Gaussian white noise added to the measured output. After a brief transient phase, the blood 

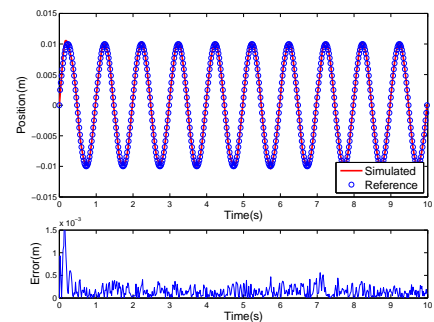

(a) Aggregate position: reference and real trajectories and tracking error.

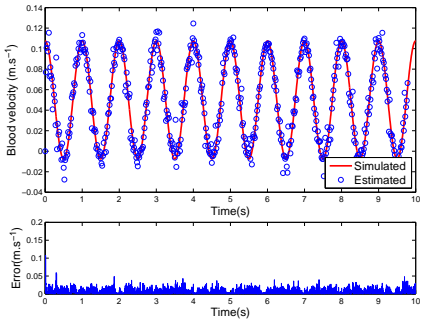

(c) Blood velocity: real and estimated velocities and estimation error.

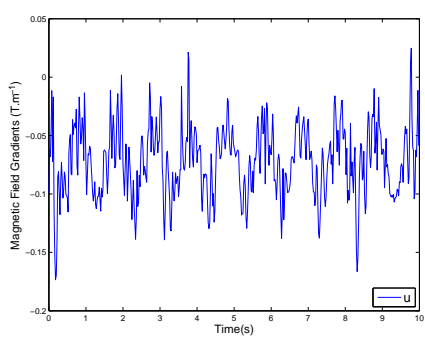

(e) Control input: $\nabla B$.

Fig. 5. Simulation with a Receding Horizon Estimation.

velocity is correctly reconstructed with an average error less than $1 m m . s^{-1}$.

\section{Simulation with a Receding Horizon Estimation}

In order to show that the efficiency of the RHE does not depend on the algorithm, we have deliberately chosen a standard algorithm (fminsearch function in Matlab based on a simplex method). The past estimation horizon is chosen equal to 5 (with a discretization period of $2 \mathrm{~ms}$ ) and the matrix $Q$ is the identity matrix. The estimated state vector converges to the simulated one although the algorithm is basic and the measured output is noised (see Figures 5(b), 5(c) and 5(d)). The control law using the estimated state feedback is able to achieve the reference trajectory tracking (see Figure 5(a)). As can be seen in the figures, the estimator needs to acquire $N_{e}$ measures before starting the optimization. This is illustrated by the variations at the beginning. Results could be largely improved by using a more sophisticated algorithm (LevenbergMarquardt) and consequently be more robust to noise and modeling errors.

\section{DISCUSSION AND CONCLUSION}

Many studies on the measurement of the average blood velocity are available in the literature. Unfortunately, this information is neither sufficient nor accurate enough for an aggregate navigating in the vasculature. The drag force has a major impact on the dynamic behavior of the system and this force strongly and nonlinearly depends on the relative velocity between the aggregate and the time-varying blood flow. The blood velocity is pulsatile and periodic. Since any periodic signal has a Fourier series decomposition, it is then possible to define a dynamic model of the blood velocity, yielding to an extending system.

The core idea of the paper was to estimate both the blood and the aggregate velocities using the sole measurement of the aggregate position given by an imager. The extended system (11) was proved to be observable and then two different observers were proposed in order to estimate the full state vector required to implement the control law. The state observer was combined with a control law based on Lyapunov functions. The simulations demonstrated the benefits to on-line estimate the blood velocity in order to ensure a precise stabilization of the aggregate of nanorobots along any reference trajectory.

The high gain observer whose convergence is theoretically well founded obtained satisfactory results. Yet the tuning of the design parameters was difficult because of their sensitivity in regard to noise and modeling errors. This phenomenon depends on the choice of the high gain $L$ and the matrix $S$. A good conditioning of the matrix $S$ is not always easy to obtain and furthermore the matrix $\Delta$ is formed in ascending powers of $L$ in (19). It is therefore necessary to make a compromise to ensure a fast convergence of the estimator with a high gain $L$ not increasing too much. The receding horizon estimator, based on a nonlinear minimization problem, was very easy to implement and provided an efficient on-line estimation but without proof of convergence. The tuning parameters were the estimation horizon $N_{e}$ and the symmetric matrix $Q$. The obtained results can largely be improved by using an efficient algorithm and also a time-varying matrix $Q$ which can play a role similar to a forgetting factor by weighting the recent measurements.

This work can be very useful in therapeutic diagnosis where the estimation of the blood velocity is a relevant information. The use of aggregates can also be considered in the case of clogged arteries with two objectives: firstly, nanorobots will remove these plaques and in a second time, the medical team can on-line check that the plaques have been correctly removed by observing the evolution of the estimated blood velocity.

Some technical limitations of this approach have to be noted. The access to hard-to-reach body's area such as capillaries requires a substantial reduction of the aggregate diameter, yet the force of propulsion and the localization of the system directly depend on its size (see Table I). The usual imagers do not have sufficient resolution and the actuators encounter power limitations for this kind of application in small vessels. Spatial resolution of the imager can also be a limitation since the noise measurement affects the quality of the blood velocity estimation. Yet preliminary results indicate that these observers are efficient even considering the modern clinical MRI or CT devices, i.e. for a $500 \mu \mathrm{m}$ resolution. 


\section{ACKNOWLEDGMENT}

The authors would like to thank the anonymous reviewers for their helpful comments that contribute to improving the quality of the paper.

\section{APPENDIX}

Let $c_{i}(t)=a_{i} \cos (i \omega t)+b_{i} \sin (i \omega t)$ and $\aleph_{1}$ be defined as a $n$th order truncated Fourier series:

$$
\aleph_{1}=a_{0}+\sum_{i=1}^{n} c_{i}(t)
$$

We show that (A.37) is the solution of the linear system (2) by induction on the following property $\forall k \in\{1, n\}$ :

$\left(P_{k}\right)\left\{\begin{aligned} \aleph_{2 k+1}= & (-1)^{k} \sum_{i=k+1}^{n}\left(\prod_{j<k+1}\left(i^{2}-j^{2}\right)\right) c_{i}(t) \\ & +a_{0} \prod_{i=1}^{k} i^{2}\end{aligned}\right.$

To show that the base case holds for $k=1$, we differentiate twice (A.37):

$$
\begin{aligned}
\ddot{\aleph}_{1} & =-\omega^{2}\left(\sum_{i=1}^{n} i^{2} c_{i}(t)\right) \\
& =-\omega^{2}\left(\aleph_{1}-\aleph_{3}\right)
\end{aligned}
$$

with $\aleph_{3}$ defined by:

$$
\aleph_{3} \triangleq a_{0}-\sum_{i=2}^{n}\left(i^{2}-1\right) c_{i}(t) .
$$

The property (A.38) consequently holds for $k=1$.

The inductive step needs to differentiate twice the expression of $\aleph_{2 k+1}$ given in (A.38):

$$
\ddot{\aleph}_{2 k+1}=-\omega^{2}\left((-1)^{k} \sum_{i=k+1}^{n}\left(\prod_{j<k+1}\left(i^{2}-j^{2}\right)\right) i^{2} c_{i}(t)\right) .
$$

From system (2), $\aleph_{2 k+3}$ is:

$$
\aleph_{2 k+3}=\frac{\ddot{\aleph}_{2 k+1}}{\omega^{2}}+(k+1)^{2} \aleph_{2 k+1}
$$

and using (A.41), we obtain:

$$
\begin{aligned}
\aleph_{2 k+3}= & -(-1)^{k} \sum_{i=k+1}^{n}\left(\prod_{j<k+1}\left(i^{2}-j^{2}\right)\right) i^{2} c_{i}(t) \\
& +\left((-1)^{k} \sum_{i=k+1}^{n}\left(\prod_{j<k+1}\left(i^{2}-j^{2}\right)\right) c_{i}(t)\right. \\
& \left.+a_{0} \prod_{i=1}^{k} i^{2}\right)(k+1)^{2} \\
= & (-1)^{k} \sum_{i=k+1}^{n}\left((k+1)^{2}-i^{2}\right) \prod_{j<k+1}\left(i^{2}-j^{2}\right) c_{i}(t) \\
& +a_{0} \prod_{i=1}^{k+1} i^{2} \\
= & (-1)^{k+1} \sum_{i=k+1}^{n} \prod_{j<k+2}\left(i^{2}-j^{2}\right) c_{i}(t) \\
& +a_{0} \prod_{i=1}^{k+1} i^{2},
\end{aligned}
$$

thereby proving that the property (A.38) holds for $k+1$.

For $k=n+1$ the property (A.38) implies that $\aleph_{2 n+1}=$ $a_{0} \prod_{i=1}^{n} i^{2}$, i.e. the mean value up to a constant factor. Using he Cauchy-Lipschitz theorem, the solution $\aleph_{1}$ is unique for a given set of initial conditions, which concludes the proof.

\section{REFERENCES}

[1] B. J. Nelson, I. K. Kaliakatsos, and J. J. Abbott, "Microrobots for minimally invasive medecine," Annual Review of Biomedical Engineering, vol. 12, pp. 55-85, 2010.

[2] B. Kristo, J. C. Liao, H. P. Neves, B. M. Churchill, C. D. Montemagno, and P. G. Schulman, "Microelectromechanical systems in urology," Urology, vol. 61(5), pp. 883-887, 2003.

[3] G. Kosa, M. Shoham, and M. Zaaroor, "Propulsion method for swimming microrobots," IEEE Transactions on Robotics, vol. 23(1), pp. 137150, 2007.

[4] W. Andr and H. Nowak, Introduction, in magnetism in medicine: a handbook (second edition). Wiley-VCH Verlag $\mathrm{GmbH} \& \mathrm{Co}$. KGaA, Weinheim, Germany, 2007.

[5] L. Zhang, J. J. Abbott, L. X. Dong, B. E. Kratochvil, D. J. Bell, and B. J. Nelson, "Artificial bacterial flagella: Fabrication and magnetic control," Applied Physics Letters, vol. 94(6), 2009.

[6] O. Ergeneman, J. J. Abbott, G. Dogangil, and B. J. Nelson, "Functionalizing intraocular microrobots with surface coatings," IEEE RAS/EMBS International Conference on Biomedical Robotics and Biomechatronics, pp. 232-237, 2008.

[7] M. C. Lagomarsino, F. Capuani, and C. P. Lowe, "A simulation study of the dynamics of a driven filament in an aristotelian fluid," Journal of Theoretical Biology, vol. 224(2), pp. 215-224, 2003.

[8] A. A. Evans and E. Lauga, "Propulsion by passive filaments and active flagella near boundaries," Physical Review E, vol. 82(4), p. 041915, 2010.

[9] R. Dreyfus, J. Beaudry, M. L. Roper, M. Fermigier, H. A. Stone, and J. Bibette, "Microscopic artificial swimmers," Nature, vol. 437, pp. 862 865, 2005.

[10] J. J. Abbott, K. E. Peyer, M. C. Lagomarsino, L. Zhang, L. X. Dong, I. K. Kaliakatsos, and B. J. Nelson, "How should microrobots swim?" International Journal of Robotics Research, vol. 28, pp. 1434-1447, 2009.

[11] J.-B. Mathieu, G. Beaudoin, and S. Martel, "Method of propulsion of a ferromagnetic core in the cardiovascular system through magnetic gradients generated by an mri system," IEEE Transactions on Biomedical Engineering, vol. 53, no. 2, pp. 292-299, 2006.

[12] S. Martel and M. Mohammadi, "Using a swarm of self-propelled natural microrobots in the form of flagellated bacteria to perform complex micro-assembly tasks," IEEE International Conference on Robotics and Automatics, pp. 500-505, 2010.

[13] P. Pouponneau, J. C. Leroux, G. Souez, L. Gaboury, and S. Martel, "Coencapsulation of magnetic nanoparticles and doxorubicin into biodegradable microcarriers for deep tissue targeting by vascular navigation," Biomaterials, vol. 32, pp. 3481-3486, 2011.

[14] P. Vartholomeos, C. Mavroidis, and N. Hata, "Simulation platform for self-assembly structures in mri-based nanorobotic drug delivery systems," IEEE International Conference on Robotics and Automation, pp. 5594 - 5600, 2010.

[15] L. Arcese, M. Fruchard, and A. Ferreira, "Endovascular magneticallyguided robots: navigation modeling and optimization," IEEE Transactions on Biomedical Engineering, vol. 59(4), pp. 977-987, 2012.

[16] P. Vartholomeos and C. Mavroidis, "In silico studies of magnetic microparticle aggregations in fluid environments for mri-guided drug delivery," IEEE Transactions on Biomedical Engineering, vol. 59(11), pp. 3028-3038, 2012.

[17] C. S. Kim, C. Kiris, D. Kwak, and T. David, "Numerical simulation of local blood flow in the carotid and cerebral arteries under altered gravity," Journal of Biomechanical Engineering, vol. 128(2), pp. 194202, 2006.

[18] Y. Rao, Y. S. Ni, and C. F. Liu, "Multi-bifurcation effect of blood flow by lattice boltzmann method," Chinese Physics Letters, vol. 25(11), pp. 4038-4041, 2008. 
[19] J. Gangloff, R. Ginhoux, M. de Mathelin, L. Soler, and J. Marescaux, "Model predictive control for compensation of cyclic organ motions in teleoperated laparoscopic surgery," IEEE Trans. on Control Systems Technology, vol. 14(2), pp. 235-246, 2006.

[20] S. Berger, W. Goldsmith, and E. Lewis, Introduction to Bioengineering. Oxford University Press, New York, 1996.

[21] R. Ponzini, C. Vergara, G. Rizzo, A. Veneziani, A. Roghi, A. Vanzulli, O. Parodi, and A. Redaelli, "Womersley number-based estimates of blood flow rate in doppler analysis: In vivo validation by means of phase-contrast mri," IEEE Transactions on Biomedical Engineering, vol. 57(7), pp. 1807-1815, 2010.

[22] K. W. Ferrara, B. G. Zagar, J. B. Sokil-Melgar, R. H. Silverman, and I. M. Aslanidis, "Estimation of blood velocity with high frequency ultrasound," IEEE Transactions on Ultrasonics, Ferroelectrics and Frequency Control, vol. 43(1), pp. 149-157, 1996.

[23] L. Arcese, M. Fruchard, and A. Ferreira, "Adaptive controller and observer for a magnetic microrobot," IEEE Transactions on Robotics, vol. 29(4), pp. 1060-1067, 2013.

[24] J. Womersley, "Method for the calculation of velocity, rate of flow and viscous drag in arteries when the pressure gradient is known," The Journal of physiology, vol. 127(3), pp. 553-563, 1955.

[25] F. White, Viscous Fluid Flow. McGraw Hill New-York, 1991.

[26] R. Kehlenbeck and R. D. Felice, "Empirical relationships for the terminal settling velocity of spheres in cylindrical columns," Chemical Eng. Technology, vol. 21, pp. 303-308, 1999.

[27] L. M. Silverman and H. E. Meadows, "Controllability and observability in time-variable linear systems," SIAM Journal on Control and Optimization, vol. 5, pp. 64-73, 1967.

[28] A. Isidori, Nonlinear control systems. Springer-Verlag London, 1995.

[29] J. Gauthier, H. Hammouri, and S. Othman, "A simple observer for nonlinear systems. application to bioreactors." IEEE Transactions on Automatic Control, vol. 37(6), pp. 875-880, 1992.

[30] H. Hammouri, B. Targui, and F. Armanet, "High gain observer based on a triangular structure," Int. J. Robust Nonlinear Control, vol. 12(6), pp. 497-518, 2002.

[31] V. M. Zavala, C. D. Laird, and L. T. Biegler, "A fast moving horizon estimation algorithm based on nonlinear programming sensitivity," Journal of Process Control, vol. 18(9), pp. 876-884, 2008.

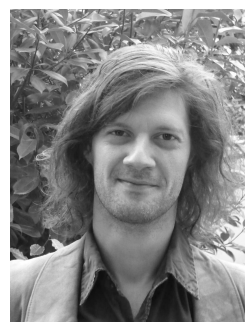

Matthieu Fruchard received the Dipl. Ing. and the $\mathrm{Ph} . \mathrm{D}$. degrees from Ecole Centrale of Lille, France, in 2001, and from the Ecole des Mines de Paris, Sophia-Antipolis, France, in 2005, respectively.

$\mathrm{He}$ joined the laboratory PRISME (Laboratoire Pluridisciplinaire de Recherche en Ingénierie des Systèmes, Mécanique et Energétique), University of Orléans, Bourges, France, in 2007, where he is currently Associate Professor. His research interests include control and observer synthesis for classes of nonlinear systems using Lyapunov theory.

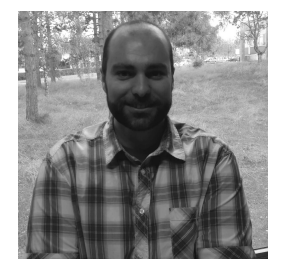

Laurent Arcese received the M.Sc. degree from the University of Lyon, France, in 2008, and the Ph.D. degree in Automatic Control from the University of Orléans, France, in 2011.

$\mathrm{He}$ is currently an Associate Professor at the Centre de Recherche en Sciences et Technologies de l'Information et de la Communication (CReSTIC), University of Reims Champagne-Ardenne, Reims, France. His research interests include the synthesis of controllers from Lyapunov theory and observer design for classes of nonlinear systems.

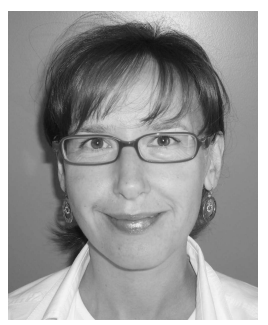

Estelle Courtial received the Ph.D. degree in Automatic Control from the University of Claude Bernard, Lyon, France.

She is currently Associate Professor at the laboratory PRISME of the University of Orléans, France. Her research interests include control and estimation for nonlinear systems with an optimization-based approach. 